\title{
A CT-scanner study of foam dynamics in porous media
}

\author{
Chakib Ouali*, Elisabeth Rosenberg, Loic Barré, and Bernard Bourbiaux \\ IFP Energies nouvelles, 1-4 avenue de Bois-Préau, 92852 Rueil-Malmaison Cedex, France
}

Received: 27 July 2018 / Accepted: 14 January 2019

\begin{abstract}
We report an experimental study of $\mathrm{N}_{2}$-foam injection in a Bentheimer sandstone coupled with X-ray Computed Tomography (CT-scanner). The measurements of pressure drop and gas saturation at different flow rates and foam qualities allowed us to describe the foam dynamics under transient and steady-state flow conditions. The brine displacement by foam shows a transient piston-like displacement pattern taking place in two successive phases. Saturation profiles reveal permanent entrance effects related to the injection procedure, and transient downstream end effects related to the gradual foam build-up. Entrance effects are attenuated with a high foam quality and at low total flow rate. The rheological behavior of foam was studied in terms of apparent foam viscosity and foamed-gas mobility as a function of foam quality and gas interstitial velocity. In the low-quality regime, foam exhibits a shear-thinning behavior that can be modelled by a power function. Furthermore, for a fixed total velocity, the quasi-invariance of strong foam apparent viscosity values is shown to result from the slight increase of trapped gas saturation, within the commonly-admitted assumption of invariant foam texture in the low-quality regime. An increase in gas mobility was observed above a certain value of the foam quality. That transition between low-quality and high-quality regimes was related to a limiting capillary pressure of foam in the porous medium under consideration.
\end{abstract}

\section{Nomenclature}

$U \mathrm{~g}, U_{\mathrm{w}}$

$Q_{\mathrm{g}}, Q_{\mathrm{w}}$

$Q_{\mathrm{t}}$

$\Delta P$

$f_{\mathrm{g}}$

$f_{\mathrm{w}}$

$S_{\mathrm{g}}, S_{\mathrm{w}}$

$S_{\mathrm{g}}^{*}, S_{\mathrm{w}}^{*}$

PVI

$P_{\mathrm{c}}$

$P_{\mathrm{c}}^{*}$

$\Phi$

A

K

$\mu_{\mathrm{g}}$

$\mu_{\text {app }}$

$v_{\text {int }_{\mathrm{g}}}$

$\mathrm{CT}_{\text {wet }}, \mathrm{CT}_{\text {dry }}$

$\rho_{\text {water }}$
Gas and water Darcy velocities

Gas and water flow rates

Total flow rate

Pressure drop

Foam quality

Water fractional flow

Gas and water saturations

Limiting gas and water saturations

Total pore volume injected

Capillary pressure

Limiting capillary pressure

Porosity

Cross section

Permeability

Gas viscosity

Foam apparent viscosity

Gas mean interstitial velocity

CT values for the core saturated with

brine and the dry core

X-ray attenuation coefficient of water $\rho(x, y, z) \quad \mathrm{X}$-ray attenuation coefficient at the voxel $(x, y, z)$

\section{Introduction}

Foam can exist in different physical states (liquid or solid) and is used in different industrial processes: food, pharmaceuticals, aquifer remediation, $\mathrm{CO}_{2}$ sequestration, etc. In petroleum engineering, liquid foam is used as a mobility control agent during gas injection to enhance sweep efficiency in oil recovery process, or to improve matrix acidizing. The efficiency of foam comes from its ability to overcome gravity segregation, viscous fingering, gas channeling and permeability heterogeneities that are gas-related problems when the latter is injected alone in the reservoir. Foam as an EOR method has been proved both in laboratory and pilot tests [1-4].

Foam flow in porous media is a widely discussed topic since the sixties and the main complexity of dealing with this process is not only the large amount of parameters that are involved but also the multiple apparent contradictory results found in the literature. A general schematic

\footnotetext{
* Corresponding author: chakib.ouali@ifp.fr
} 
description of foam in porous media was given by Radke and Gillis [5], where liquid containing surfactants form thin films called "lamellae" and separate gas bubbles (either in movement or in a trapped state) (Fig. 1). Other studies describe the general behavior of foams in porous media; Osterloh and Jante [6] conducted several coreflood experiments in sandpacks and showed the influence of liquid and gas velocity on the pressure drop of $\mathrm{N}_{2}$ foam. They dissociated two regimes as a function of foam quality (gas volume fraction): low-quality and high-quality regimes. In the first regime, foam would be characterized by intermittent advance and apparent trapping of bubbles with a pressure gradient that is nearly independent of liquid superficial velocity $U_{\mathrm{w}}$. The second regime would be rather characterized by bubble coalescence, with a pressure gradient that is nearly independent of gas superficial velocity $U_{\mathrm{g}}$ [7]. The transition from the first to the second regime occurs for a critical foam quality called " $f_{\mathrm{g}}^{*}$ " where the pressure drop is maximum and foam bubbles start to coalesce. That coalescence threshold corresponds to the limit of stability of lamellae. The latter is reached when the difference between gas phase pressure within bubbles and water phase pressure within lamellae, i.e. the gas-water capillary pressure, balances the intermolecular forces that are responsible for film stability [8]. That is, the foam coalescence is initiated when a critical or limiting capillary pressure, denoted " $P_{\mathrm{c}}^{* \text { ", }}$, is reached, or when the liquid phase saturation of the porous medium falls below the critical value corresponding to " $P_{\mathrm{c}}^{*}$ " $[8,9]$.

Several parameters are believed to affect foam flow in porous media [10]. The type of gas [11-14], surfactant formulation [15], pressure gradient [16], entrance effects [17] and porous medium geometry $[18,19]$ and permeability [20-23] are some of the parameters studied to describe foam rheological behavior.

The decrease of gas mobility when flowing as a foam in a porous medium can be seen either as an increase of gas viscosity $[18,24]$ or as a decrease of relative permeability to gas $[25,26]$. On one hand, it was demonstrated that foam behaves as a shear thinning fluid in capillaries and that the viscosity is a linear function of the density of lamellae (texture) [24, 27]. The increase of texture induces a high resistance to gas flow thus decreasing the gas mobility. On the other hand, foamed gas trapping reduces the number of flow paths for gas transport in the porous medium [28]. Besides, foam seems to behave as a yield stress fluid and thus a minimum velocity (or minimum pressure gradient) is needed to mobilize/generate "strong" foams [29-31].

While recent microfluidic studies revealed important mechanisms of foam flow as bubble fragmentation processes [32] and preferential flow paths and local flow intermittency [33], X-ray Computed Tomography (CT) experiments remain experiments of choice when dealing with consolidated cores to visualize and quantify in-situ local gas and liquid saturations during foam flow.

In this paper, we present a series of $\mathrm{N}_{2}$-foam injection experiments in a Bentheimer sandstone at different velocities coupled with CT measurements. Previous literature works [34-40] have enabled foam flow dynamics visualization by X-ray tomography. However, none of them presented an extensive CT-scanner study of foam flow at

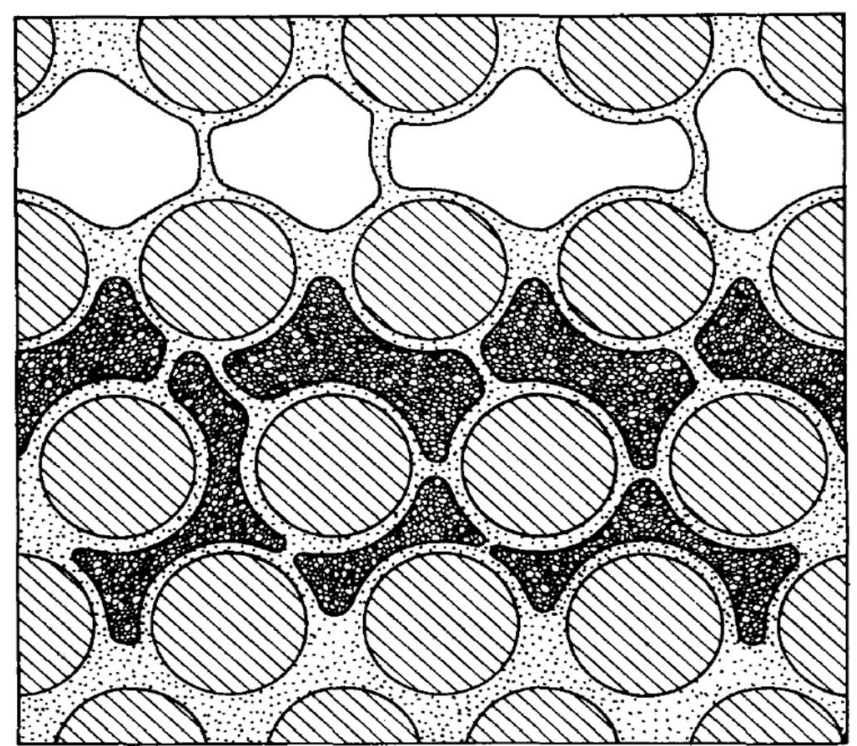

Fig. 1. A schematic of foam flow at the pore scale (from reference [5]). Flowing bubbles are unshaded, and trapped gas is darkly shaded. The grains are represented by circles.

different velocities and foam qualities in relation with the porous medium microscopic features. After describing the foam dynamics in the first set of results, we describe the rheological behavior of the foam in terms of foam viscosity and foamed gas mobility. The transition between the low-quality and the high-quality regimes is quantified in terms of limiting capillary pressure (as extracted by mercury porosimetry) that triggers foam coalescence.

\section{Materials and experiments}

\subsection{Experimental set-up}

The experimental set-up is shown in Figure 2. The masterpiece is an aluminum-made coreholder of "Hassler" type. The tips of the coreholder are made with PEEK. The core is mounted inside a sheath made of Perlast ${ }^{\circledR}$ fluoroelastomer that does not much attenuate X-rays. A high-salinity brine $(\mathrm{NaCl} 4 \mathrm{wt} \%)$ is injected with a Pharmacia pump (P-500) and the surfactant aqueous solution (a mixture of two surfactants, $0.1 \mathrm{wt} \%$ each in the same $\mathrm{NaCl}$ brine) is injected with a Vindum Pump (VP-series). The nitrogen gas injection is performed with Brooks gas mass flow meters. Two gas mass flow meters were used depending on the gas flow rate target. The model SLA5850S working in the range $(0-180 \mathrm{~mL} / \mathrm{h})$ at $0{ }^{\circ} \mathrm{C}$ and 1 bar was used for low flow rates, and the model $5850 \mathrm{E}$ working in the range $(0-3 \mathrm{~L} / \mathrm{h})$ at $0{ }^{\circ} \mathrm{C}$ and $1 \mathrm{bar}$ for high flow rates. The baseline pore pressure was fixed by a Back Pressure Regulator (BPR) (model SLA5820B) at 10 bar. The gas mass flow meter and the BPR were connected to a Brooks Microprocessor Control \& Read Out Unit model 0152, which allows to change set points.

To follow pressure measurements, four Keller pressure transducers were used; three absolute transducers 


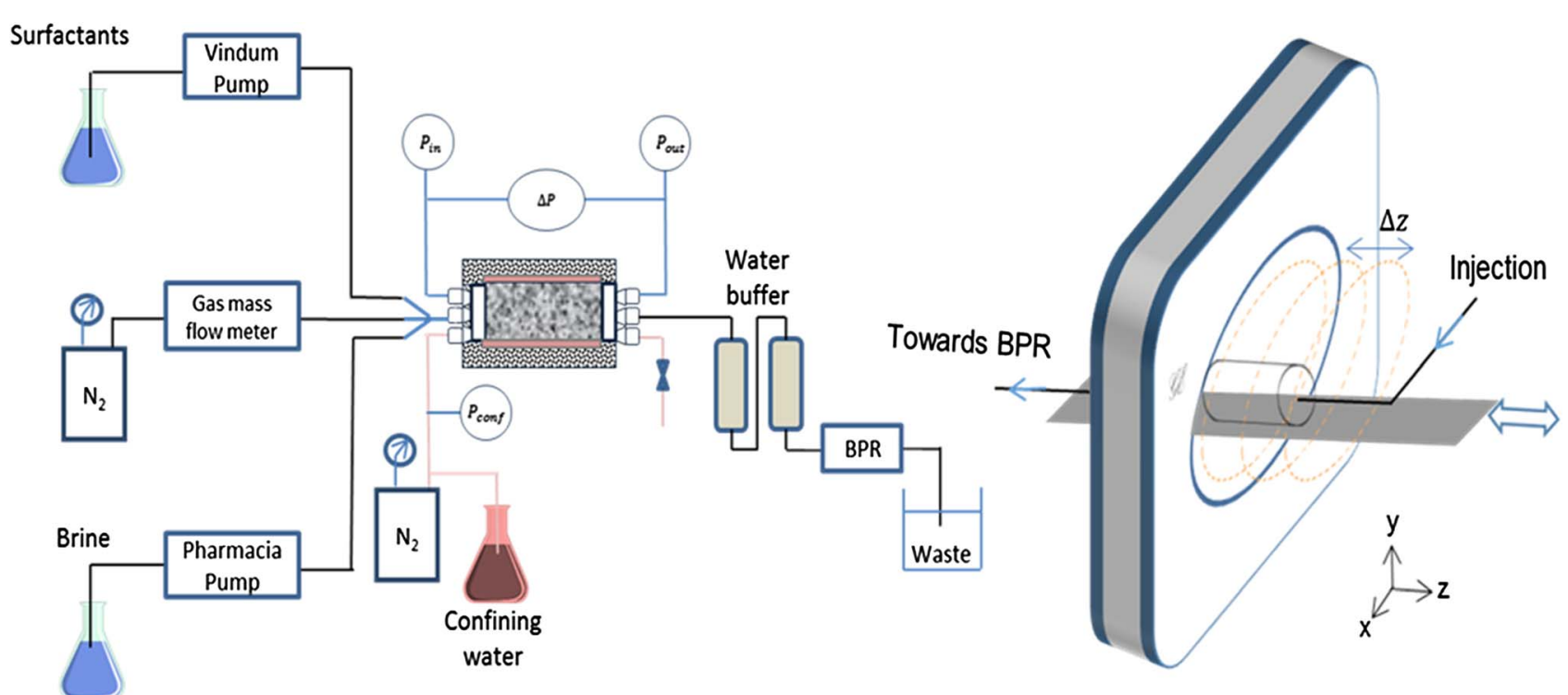

Fig. 2. On the left, the experimental setup to generate foam in the porous medium. On the right, a schematic of the X-ray CT scanner with the core in the gantry.

(model PA-33X) and a differential one (model PD-39X). We used home developed software based on Labview to read and record pressures in real time. Pure water is used as a confining liquid, while the confining pressure (between 20 bar and 30 bar) is maintained by pressurized nitrogen reservoir. The control of this pressure is realized with a pressure gauge mounted on the pressurized nitrogen gas bottle.

In this experimental set-up we used two tanks of $1 \mathrm{~L}$ capacity each, between the core outlet and the BPR. These reservoirs are filled with pure water allowing the separation of gas and liquid from the foam exiting the core. Thanks to this arrangement, a stable pressure could be maintained within the core because only water was flowing through the BPR, thus avoiding the uncontrollable rate/pressure fluctuations occurring when a gas-liquid flow is regulated.

\subsection{CT-scanner apparatus}

The CT-scanner used in this study is General Electric GE Discovery $750 H D$ Dual Energy. The core is mounted horizontally on the table of the scanner as shown in Figure 2. During the displacement of the table, the couple sourcedetectors rotate around the core in a synchronized way to form an image of the attenuated X-rays. More information on the use of the CT-scanner to form tomographic images can be found elsewhere [41, 42].

We obtain a stack of joined $1.25 \mathrm{~mm}$ thick slices with a pixel size of $(117 \mu \mathrm{m}, 117 \mu \mathrm{m})$ in the $(x, y)$ plane. Table 1 summarizes the image acquisition settings.

For any voxel (volume element of sample), the scanner measures the linear attenuation coefficient of X-rays, which is expressed in CT units of Hounsfield scale as follows:

$$
\mathrm{CT}=1000\left(\frac{\rho(x, y, z)}{\rho_{\text {water }}}-1\right)
$$

where $\rho(x, y, z)$ is the linear attenuation coefficient at the voxel position of co-ordinates $(x, y, z)$ and $\rho_{\text {water }}$ is the linear attenuation coefficient of water. The linear attenuation coefficients are calculated from the Beer-Lambert's law applicable to the quasi-monochromatic X-ray beam crossing the sample.

For a two-phase flow in the core, the gas and water saturations, $S_{\mathrm{g}}$ and $S_{\mathrm{w}}$, are determined from the recorded $\mathrm{CT}$ value by using the following equations:

$$
S_{\mathrm{g}}=\frac{\mathrm{CT}_{\text {wet }}-\mathrm{CT}}{\mathrm{CT}_{\text {wet }}-\mathrm{CT}_{\text {dry }}},
$$

and

$$
S_{\mathrm{w}}=1-S_{\mathrm{g}},
$$

where $\mathrm{CT}_{\text {wet }}$ and $\mathrm{CT}_{\text {dry }}$ are $\mathrm{CT}$ values for the core saturated with brine and the dry core. The estimated error on saturation is about 0.01 .

\subsection{Description of the porous medium}

A Bentheimer sandstone, $4.95 \mathrm{~cm}$ length and $2 \mathrm{~cm}$ diameter, was used to carry out the experiments. This type of sandstone is homogeneous, mainly made of quartz $(>95 \%)$. The porosity profile measured by the CT scanner along the core is shown in Figure 3. The core has a mean porosity of $24.7 \%$, and a mean single-phase permeability to brine of $2.17 \pm 0.12 \mathrm{D}$.

The gas-water capillary pressure curve of that porous medium is shown in Figure 4. That curve, which refers to a drainage displacement process (non-wetting phase, gas, displacing wetting phase, water), was inferred from a mercury injection curve measured on a sister plug extracted from the same block of Bentheimer sandstone. Transcription of mercury $P_{\mathrm{c}}$ curve to a gas-water $P_{\mathrm{c}}$ curve was 
Table 1. Main properties of the X-ray CT scanner.

\begin{tabular}{lc}
\hline Specification & Quantity \\
\hline Tube voltage & $140 \mathrm{kV}$ \\
Tube current & $250 \mathrm{~mA}$ \\
Slice thickness $(\Delta z)$ & $1.25 \mathrm{~mm}$ \\
Scan time & $5.36 \mathrm{~s}$ \\
Number of slices/scan & 41 \\
Field of view & $6 \mathrm{~cm}$ \\
Voxel size $(x, y, z)$ & $(117 \mu \mathrm{m}, 117 \mu \mathrm{m}, 1.25 \mathrm{~mm})$ \\
\hline
\end{tabular}

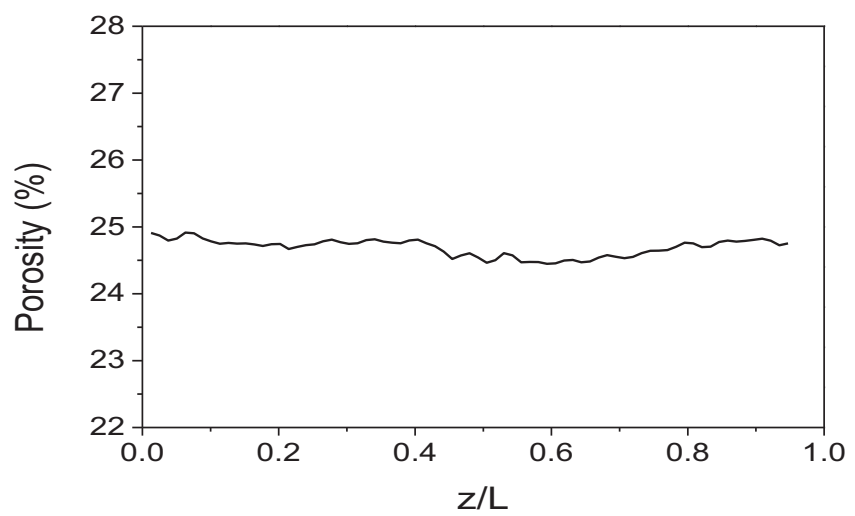

Fig. 3. Porosity profile of the Bentheimer core.

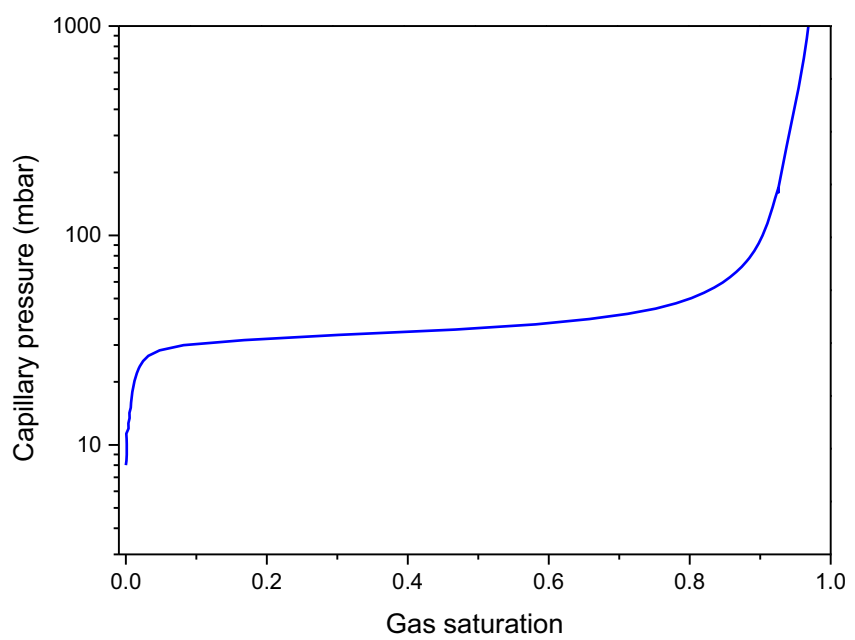

Fig. 4. Gas-water capillary pressure as a function of gas saturation.

performed by using Young-Laplace equation with an interfacial tension of $28.8 \mathrm{mN} / \mathrm{m}$ between air and surfactant solution.

\subsection{Coreflood procedure}

After placing the core inside the coreholder and applying a confining pressure, a first scan of the dry core is realized,
Table 2. Experimental parameters for gas and surfactant co-injection.

\begin{tabular}{ccccc}
\hline Exp & $Q_{\mathrm{t}}(\mathrm{mL} / \mathrm{h})$ & $\mathrm{Q}_{\mathrm{g}}(\mathrm{mL} / \mathrm{h})$ & $Q_{\mathrm{l}}(\mathrm{mL} / \mathrm{h})$ & Injected $f_{\mathrm{g}}$ \\
\hline 1 & 4.89 & 2.44 & 2.45 & 0.5 \\
& 4.88 & 1.95 & 2.93 & 0.6 \\
& 4.88 & 1.46 & 3.42 & 0.7 \\
& 4.88 & 0.98 & 3.9 & 0.8 \\
& 4.88 & 0.49 & 4.39 & 0.9 \\
2 & 9.76 & 4.88 & 4.88 & 0.5 \\
& 9.76 & 5.86 & 3.9 & 0.6 \\
& 9.76 & 6.83 & 2.93 & 0.7 \\
& 9.75 & 7.8 & 1.95 & 0.8 \\
& 9.75 & 8.77 & 0.98 & 0.9 \\
& 9.76 & 6.83 & 2.93 & 0.7 \\
& 9.76 & 4.88 & 4.88 & 0.5 \\
3 & 14.64 & 7.32 & 7.32 & 0.5 \\
& 14.64 & 8.78 & 5.86 & 0.6 \\
& 14.64 & 10.25 & 4.39 & 0.7 \\
& 14.64 & 11.71 & 2.93 & 0.8 \\
& 14.64 & 13.18 & 1.46 & 0.9 \\
& 14.64 & 10.25 & 4.39 & 0.7 \\
& 14.64 & 7.32 & 7.32 & 0.5 \\
& 75.33 & 30.13 & 45.2 & 0.4 \\
& 75.2 & 45.2 & 30 & 0.6 \\
\hline & 75.7 & 52.7 & 23 & 0.7 \\
& 74.8 & 67.8 & 7 & 0.91 \\
\hline & & & &
\end{tabular}

and then the core is saturated with brine at a pressure of 1 bar. The latter is increased to 10 bar and 100 pore volumes of brine are injected. An experiment consists of a co-injection of gas and surfactant solution at a fixed total flow rate $Q_{\mathrm{t}}$ and at different successive foam qualities $f_{\mathrm{g}}$ (from 0.5 to 0.9 ). The foam quality is defined as:

$$
f_{\mathrm{g}}=\frac{Q_{\mathrm{g}}}{Q_{\mathrm{g}}+Q_{\mathrm{w}}},
$$

where $Q_{\mathrm{w}}$ is the liquid flow rate and $Q_{\mathrm{g}}$ is the gas flow rate. $f_{\mathrm{g}}$ is changed after reaching the steady state, as indicated by the stability of the pressure drop. In this study, the compressibility effects are taken into account by calculating the foam quality at the average pressure between core inlet and outlet. A set of experiments was realized at different total flow rates, from $4.9 \mathrm{~mL} / \mathrm{h}$ to $75.7 \mathrm{~mL} / \mathrm{h}$ (i.e. total Darcy velocities between $1.2 \mathrm{ft} / \mathrm{d}$ and $19.2 \mathrm{ft} / \mathrm{d}$ ). Each experiment consists in a series of injections at a fixed total flow rate of gas and solution with increasing quality (Tab. 2). Additionally, for Experiments 2 and 3, foam quality was decreased after reaching the maximum $f_{\mathrm{g}}$ value of 0.9 , to test possible hysteresis effects.

At the end of each experiment, the core is placed vertically and is flushed from bottom to top with brine at different backpressures and different velocities for several 
hours, in order to remove the foam in place through a gravity-stable displacement. A scan of the core saturated with brine is realized at the end, to compare the initial and final state after each experiment. CT values of each slice did not change by more than $0.2 \%$ from one experiment to another, both for the dry and saturated core. Therefore the recovery of the core after each experiment was satisfactory regarding both initial and final saturation states. The experimental conditions are summarized in Table 2.

\section{Results and discussion}

\subsection{Description of foam dynamics}

\subsubsection{Weak foam displacement phase}

An example of gas and surfactant co-injection at $f_{\mathrm{g}} 0.5$ is presented in Figure 6. As soon as the surfactant solution and the gas are co-injected, a sharp front is formed and pushes the liquid towards the outlet in a piston-like manner. The foam quality injected is 0.5 , hence two pore volumes have to be injected for the foamed gas front to reach the core outlet $\left(\mathrm{PVI}=\frac{Q_{\mathrm{t}} t}{\mathrm{PV}}\right)($ Fig. 6$)$. The average gas saturation of the total core increases significantly as soon as the co-injection starts, with a rate of increase becoming faster after the injection of about one PV (Fig. 5a). The pressure drop remains also fairly low at that initial stage of injection, and starts to increase significantly after, concomitantly with the increase of the average gas saturation of the downstream half of the core (Fig. 5a). However, a foam is already formed and is mobilizing in situ liquid (Fig. 6) during this initial stage, as revealed also by the measured pressure drop (241 mbar in average) that is about 74 times the pressure drop measured with liquid alone (3.24 mbar) at the same flow rate. Thus viscous forces are sufficient to mobilize the liquid ahead, even if this stage would characterize a "weak foam".

The detailed evolution of saturation along the core with injected volume (Fig. 6) has to be interpreted further as follows:

\subsubsection{Entrance effects}

The water saturation at core entrance remains high and gradually decreases with the number of injected PVs, as it does also with increasing foam quality (Fig. 7) and decreasing total flow rate (Fig. 8): this "entrance" effect may be ascribed to the foam generation process that takes place within the porous medium because gas and surfactant solution feed upstream core end as separate phases. Several studies actually found that if foam is not pre-generated, the entrance region is characterized by a high liquid saturation $[39,43]$ and a low pressure gradient $[44,45]$ because the minimum pressure gradient for strong foam generation is not attained in that region. The foam at the entrance region results from the interplay between mechanisms of generation and destruction of lamellae that make foam totally different from the one in the downstream region [17]. That entrance effect was observed in all our experiments, however, it was reduced at lower total flow rates (Fig. 8). So finally, the steady-state flow of a strong "textured" foam
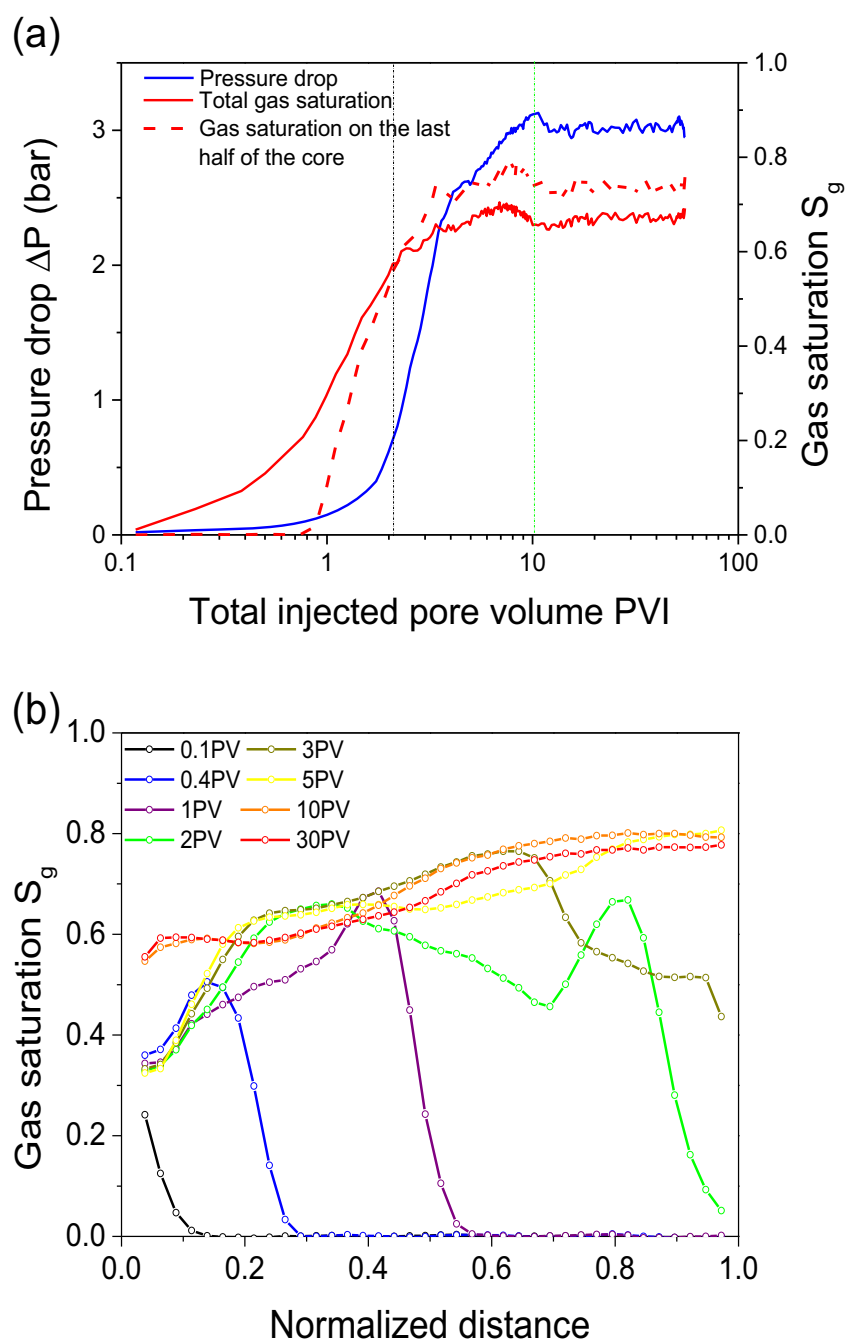

Fig. 5. (a) Evolution of pressure drop and gas saturation with the total pore volume injected and (b) gas saturation distribution within the core (for Experiment 3 at $f_{\mathrm{g}}=0.5$ ).

can be obtained but at a certain distance from core inlet, which coincided more or less with the mid-distance between inlet and outlet in our experiments.

\subsubsection{Capillary end effects}

CT scans also show a liquid retention near core outlet during the first $3 \mathrm{PV}$ of injection (Fig. 5b): this is a classical capillary end effect observed during a drainage process, that is the displacement of a wetting fluid by a non-wetting fluid. The wetting fluid is retained at the downstream end of the porous medium because gas and water pressures are equal on both sides of the downstream core boundary (i.e. capillary pressure is nil at core boundary) which implies liquid accumulation within the porous medium near core outlet. Present flow situation is somewhat different as both liquid and gas are injected and assumed to form foam within the porous medium. Nevertheless, the process remains a drainage process as the saturation of the non-wetting phase (gas) is increasing in the displacement under consideration, 


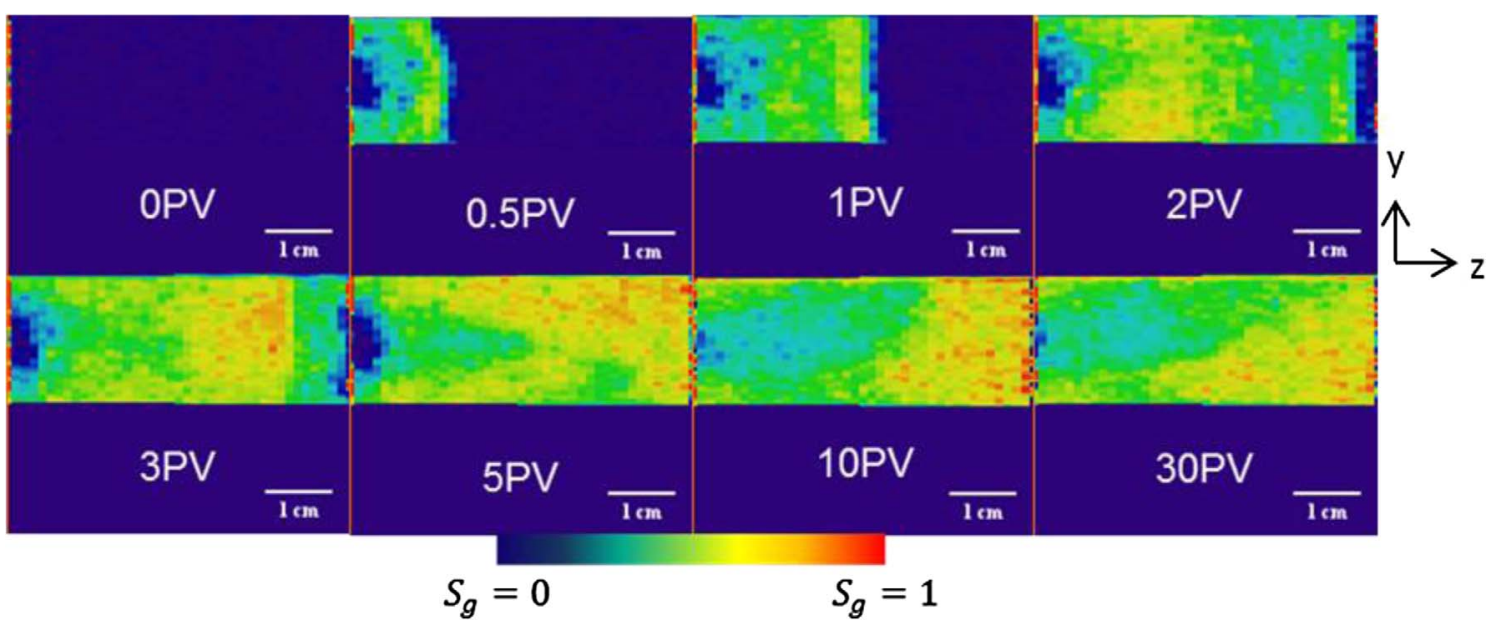

Fig. 6. The displacement of foam (green/red) in a Bentheimer sandstone core, initially saturated with brine (blue). The surfactant and Nitrogen are directly co-injected in the core at $Q_{\mathrm{w}}=7.3 \mathrm{~mL} / \mathrm{h}$ and $Q_{\mathrm{g}}=7.3 \mathrm{~mL} / \mathrm{h}$.

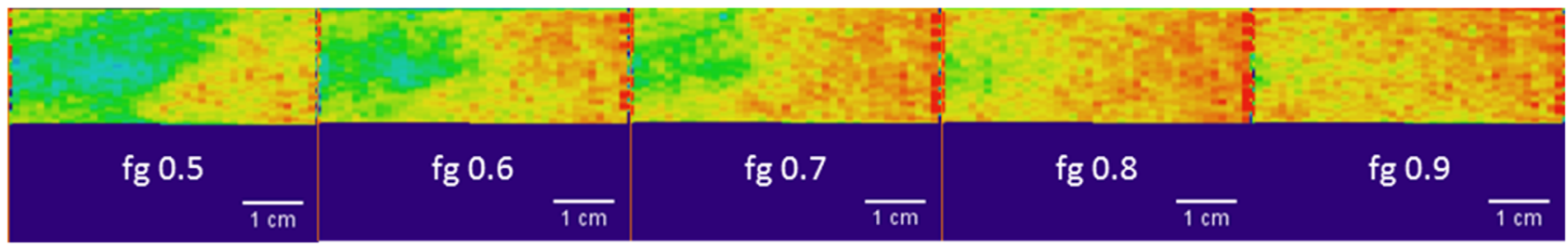

Fig. 7. Entrance effects as a function of foam quality at steady state for Experiment 2.

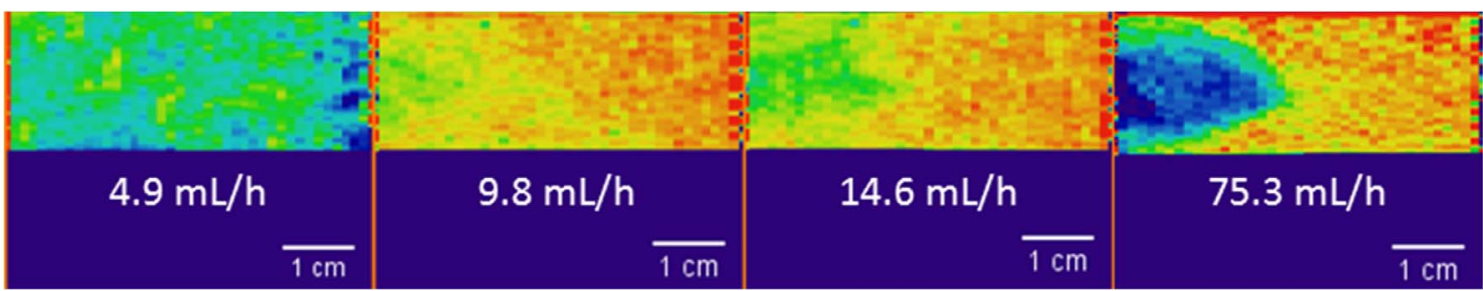

Fig. 8. Entrance effects at $f_{\mathrm{g}}=0.7$ for different total flow rates at steady state.

hence liquid retention at core outlet may be interpreted as a capillary end effect from that viewpoint. However, water saturation decreases and the end effect vanishes after the injection of many pore volumes, leading finally to the reverse saturation distribution where gas saturation is maximum at core outlet (Figs. 5b and 6). The overall evolution of saturation can finally be interpreted as a transient flow behavior between (1) an initial drainage by gas as a weak foam with few films and bubbles, hence yielding water capillary retention at core outlet, and (2) the steady-state flow of a strong foam as a single viscous phase, with a mobility ratio between foam and liquid in place that favors liquid production at core outlet.

Whereas capillary outlet end effect vanishes completely after a few PV (5 PVs on Figs. 5b and 6), the "entrance" effect is maintained even after the injection of a large number of PVs, although it is attenuated. Actually, according to previous analysis, the "entrance" effect is related to the injection process whereas the downstream end effect is related to a transient behavior of a foam that acquires its final texture within the heart of the porous medium (ahead of the upstream end) through the circulation of several PVs.

\subsubsection{Strong foam build-up phase}

In the displayed experiment, the transition from a weak foam to a strong foam occurs after the injection of $2 \mathrm{PVs}$ as indicated by the sharp increase of the pressure drop (Fig. 5a). That transition is less apparent on gas saturation profiles (Fig. 5b), although a second front is seen sweeping again the core (Fig. 6 at $3 \mathrm{PVs}$ ) after the breakthrough of the first front occurring after 2 PVs (Figs. 5 and 6). This transition characterized by a sharp increase of the pressure drop and correlated to a slight increase of the gas saturation was described by Nguyen et al. [46] as an initial phase where a coarsely-textured "weak foam" is propagated through the 
large pores, and a secondary liquid desaturation phase, where the foam invades smaller pores containing liquids and acquires a fine texture. Figure 7 shows that the capillary end effect decreases as the foam quality increases, which is consistent with the increased displacement efficiency of strong high-quality foams. Indeed, two-phase flow behavior implies that liquid saturation decreases when the fractional flow of gas increases and that liquid saturation decrease is more pronounced in the presence of a foaming fluid system where gas phase mobility is reduced [47].

\subsection{Rheological behavior of foam at steady state}

The main feature of foam displacement in a porous medium is the drastic reduction of foamed-gas mobility by comparison with gas flowing as a continuous phase. That mobility reduction of gas is quantified in flow experiments either by the pressure drop between core ends or by the apparent viscosity of foam considered as a single phase, or also by the absolute mobility of foamed-gas in the two-phase flow of foamed-gas and liquid. Foam mobility is determined under steady-state flow conditions, i.e. at the end of foam displacement. Foamed-gas mobility reduction depends on the quality of foam, i.e. the gas fractional flow, and on the flow velocity. Before discussing such results, the expressions of foam apparent viscosity and foamed-gas absolute mobility are recalled as functions of measured flow data.

Considering the foam as a single fluid, Darcy's equation allows one to define the foam apparent viscosity as:

$$
\mu_{\text {app }}=\frac{K A \Delta P}{L Q_{\mathrm{t}}},
$$

where $K, A$ and $L$ represent the permeability, the cross section and the length of the core respectively. $\Delta P$ and $Q_{\mathrm{t}}$ are respectively the pressure drop and the total flow rate.

The gas interstitial velocity is defined as:

$$
v_{\text {int }}=\frac{Q_{\mathrm{g}}}{\Phi A S_{\mathrm{g}}},
$$

where $Q_{\mathrm{g}}$ is the gas flow rate, $\Phi$ is the porosity and $S_{\mathrm{g}}$ is the mean gas saturation.

From equations (4) and (5) we can express the foam apparent viscosity as a function of the foam quality as:

$$
\mu_{\text {app }}=\frac{K A \Delta P}{L Q_{\mathrm{g}}} f_{\mathrm{g}} .
$$

And from equations (6) and (7) we can express the foam apparent viscosity as a function of the gas interstitial velocity as:

$$
\mu_{\text {app }}=\frac{K \Delta P f_{\mathrm{g}}}{L \Phi S_{\mathrm{g}}} \frac{1}{v_{\text {int }_{\mathrm{g}}}} .
$$

Considering foam displacement as a two-phase flow of foamed gas and liquid, the flow rate of foamed-gas can be expressed from generalized Darcy law as:

$$
Q_{\mathrm{g}}=\frac{K M_{\mathrm{g}}^{\mathrm{f}} A \Delta P}{L}
$$

where $M_{\sigma}^{\mathrm{f}}$ is the relative mobility of gas phase when flowing as a discontinuous phase in a foam (i.e. as a foamedgas). The relative mobility of a phase is defined as the ratio between its relative permeability and its viscosity. Regarding foam flow, the lowered relative mobility of foamed-gas can be seen as a gas relative permeability decrease or as a gas viscosity increase. Herein, to avoid any confusion and for the sake of clarity, we keep $M_{\mathrm{g}}^{\mathrm{f}}$ in its lumped form.

The absolute mobility of foamed-gas is then expressed as:

$$
K M_{\mathrm{g}}^{\mathrm{f}}=\frac{Q_{g} L}{A \Delta P}
$$

or also as:

$$
K M_{\mathrm{g}}^{\mathrm{f}}=\frac{K}{\mu_{\text {app }}} f_{\mathrm{g}} .
$$

\subsubsection{Analysis of pressure drop and foam apparent viscosity data}

The evolution of pressure drop measured at steady state is shown in Figure 9 for the four experiments at different flow rates. At fixed foam quality, the pressure drop increases as a function of total flow rate. Also, the pressure drop increases slightly with foam quality in the low-quality regime then decreases significantly at high foam qualities (high-quality regime). The transition between the two regimes occurs gradually on a small range of foam qualities around a critical foam quality $f_{\mathrm{g}}^{*}$ estimated at 0.7 . For Experiment 1, no strong foam was observed even after 60 pore volumes of foam injection. In comparison to the other experiments at relatively high flow rates, the low pressure drop measured at $4.9 \mathrm{~mL} / \mathrm{h}$ indicates that gas and liquid must be injected at a sufficient velocity to develop a strong foam. In other words, a minimum pressure gradient must be applied in order to divide bubbles and create enough lamellae and so to increase foam texture [29-31]. For that experiment, the gas saturation was low, around 0.45 for all foam qualities tested, by comparison with the gas saturation (0.8 on average) measured in other experiments at higher velocities (Fig. 13a), and no secondary liquid desaturation phase was observed. Therefore, results of Experiment 1 are not representative of the typical foam flow behavior observed in our set of experiments; hence will not much be referred to in the following.

Foam apparent viscosity is shown as a function of foam quality in Figure 10. Foam apparent viscosity is more or less constant, for foam qualities less than the critical value of 0.7 . The transition to a high-quality regime is more visible for the experiments at $9.8 \mathrm{~mL} / \mathrm{h}$ or $14.6 \mathrm{~mL} / \mathrm{h}$ than for other experiments. For the highest velocity at $75.3 \mathrm{~mL} / \mathrm{h}$, we do not observe a large difference between foam apparent viscosities in the low- and high-quality regimes.

Figures 9 and 10 reveal respectively similar pressure drop values and similar foam apparent viscosities at $f_{\mathrm{g}}$ values of 0.7 and 0.5 in a sequence of increasing then decreasing foam quality. That is, no hysteresis effects are observed as a function of foam quality. The hysteresis 


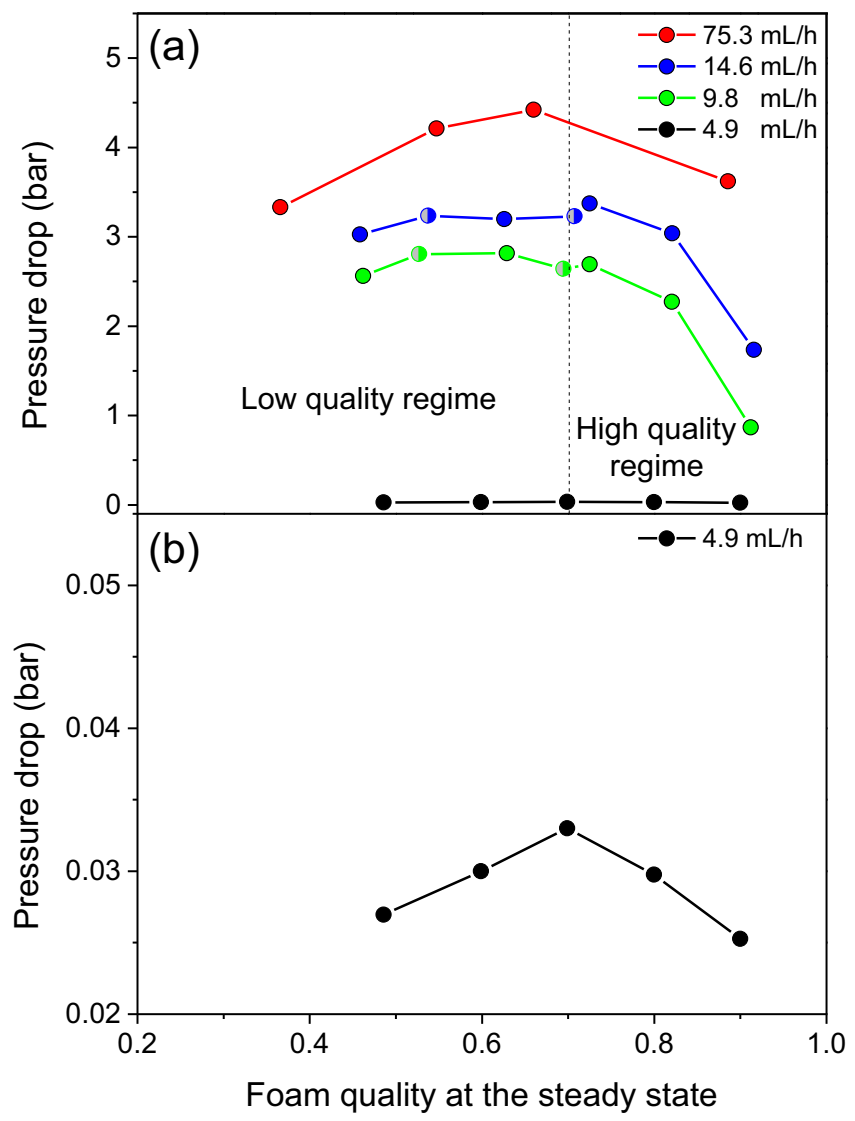

Fig. 9. (a) Pressure drop as a function of foam quality at steady state for different total flow rates. The half colored circles represent the experiments of low-quality foam after reaching the highest foam quality. (b) Zoom on the experiment at the lowest total flow rate.

effects are usually investigated by changing the total velocity for a given foam quality [48]. In our study, they are tested by changing the foam quality at a given total flow rate. The absence of any hysteresis effects herein indicates that the coarsened foam at a high-quality regime (above $f_{\mathrm{g}}^{*}$ ) can turn again into a strong finely-textured foam by decreasing the foam quality.

\subsubsection{Velocity effects}

To study the rheological behavior of foam, the foam apparent viscosity is plotted as a function of gas interstitial velocity in Figure 11 where we dissociate the low-quality and the high-quality data. The foam behaves as a shear thinning fluid if we consider only the low-quality regime $\left(\mu_{\text {app }}\right.$ decreases as a function of $\left.v_{\text {intg }}\right)$. The strong foam data in the low-quality regime can be tuned to a power function of interstitial gas velocity:

$$
\mu_{\text {app }}=\mu_{\mathrm{g}}+\alpha\left(v_{\text {intg }}\right)^{-\gamma} \approx \alpha\left(v_{\text {intg }}\right)^{-\gamma},
$$

where $\alpha=2.07 \times 10^{5}$ and $\gamma=0.83$ apply for our measurements and $\mu_{\mathrm{g}}=1.76 \times 10^{-2} \mathrm{cP}$ is the $\mathrm{N}_{2}$ gas viscosity (at $20^{\circ} \mathrm{C}$ and 1 bar). That formula takes into account the fact that the dependence of the apparent viscosity on the

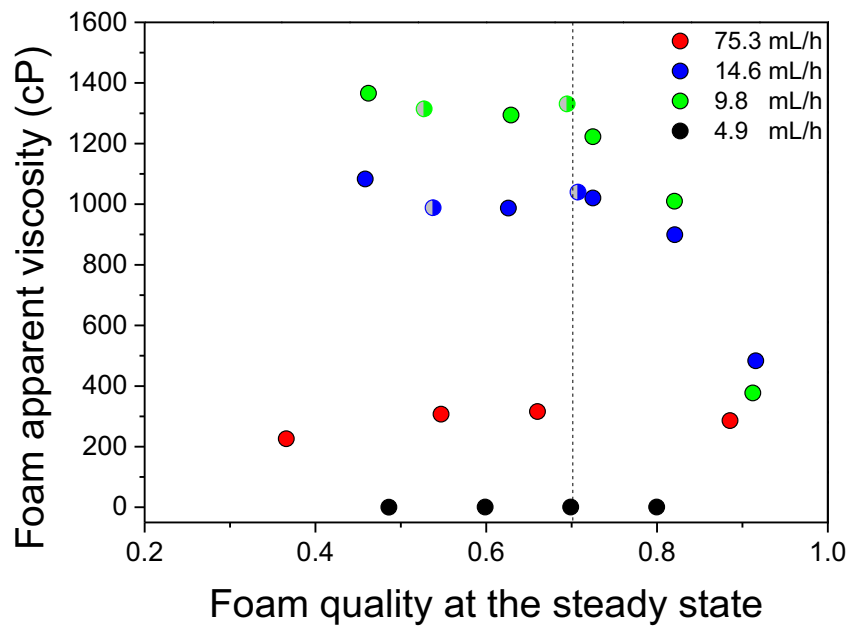

Fig. 10. Apparent foam viscosity as a function of foam quality at steady state. The half colored circles represent the experiments of low-quality foam after reaching the highest foam quality.

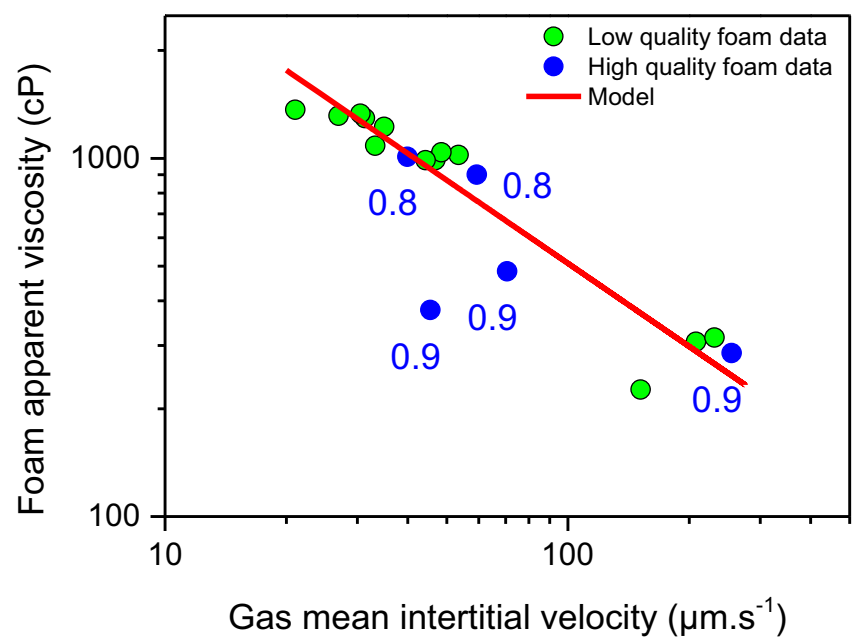

Fig. 11. A log-log representation of apparent foam viscosity as a function of the gas mean interstitial velocity for strong foam data (the curve represents a fit of low-quality data). The labels represent foam qualities for high quality foam data.

liquid flow rate is negligible. This model is similar to the model proposed by Hirasaki and Lawson [24] and other authors $[18,24,25]$, who showed that foam apparent viscosity in a capillary varied quasi-linearly with foam texture, and with the inverse of a power function of gas bubbles velocity (or more exactly lamellae velocity) [27] as:

$$
\mu_{\text {app }} \approx \frac{\beta n_{\mathrm{f}}}{v_{\text {intg }_{\mathrm{g}}}^{\frac{1}{3}}}
$$

where $n_{\mathrm{f}}$ is the density of mobile lamellas and $\beta$ is a coefficient depending on surfactant composition and concentration. Depending on the porous medium, the shear thinning exponent may range from $1 / 3$ to $1[18,24,49]$. 
In the low-quality regime $\left(f_{\mathrm{g}}<f_{\mathrm{g}}^{*}\right)$, the bubble size (i.e. the foam texture) does not change with quality and is determined by the petrophysical properties of the rock under consideration [50]. Hence the foamed-gas viscosity is only impacted by the flow velocity, according to studies on capillaries evoked before, and the magnitude of foam apparent viscosity is determined by the extent of gas bubbles trapping [7, 49].

The high-quality foam data, above $f_{\mathrm{g}}^{*}$, in contrary, seem to be dispersed around the velocity-dependent shearthinning model. Actually, the coalescence regime is more complex than the low-quality regime because the former regime involves both a change in the bubble size and a thinning of lamellae with increasing flow velocity. Furthermore, bubbles of contrasted sizes are generated through coalescence phenomenon, which induces gas diffusion through liquid films and maybe a higher sensitivity of foam texture to gas velocity [51].

\subsubsection{Foamed gas mobility}

Foam effects on displacement efficiency are usually quantified by the reduction of gas mobility when flowing as a foam instead of a continuous gas phase [52]. Figure 12 reports all absolute gas mobility data determined in all our foam flow experiments and synthetizes previously-discussed foam flow features. First, Experiment 1 data points clearly that gas mobility is much higher when a weak foam or no foam is generated instead of a strong foam as in other experiments at high total flow velocities. Second, data points for Experiments 2, 3 and 4 show the effect of flow velocity on foamed-gas mobility. Third, the increase of foamed-gas mobility at high gas saturation for Experiments 2 or 3 quantifies the effects of coalescence on foamed-gas mobility in the high-quality regime (as shown by arrows in Fig. 12).

\subsection{Effect of the porous medium on foam coalescence}

Figures $13 \mathrm{a}$ and $13 \mathrm{~b}$ show the mean foam quality $f_{\mathrm{g}}$ at steady state as a function of the average gas saturation of the downstream half of the core. Latter precaution is taken to avoid entrance effects.

\subsubsection{Low-quality regime}

For all experiments, the overall first-order trend is a very slight increase of $S_{\mathrm{g}}$ when $f_{\mathrm{g}}$ increases from 0.4 to $0.7-0.8$, i.e. in the low-quality regime. Whereas Experiment 1 shows low $S_{\mathrm{g}}$ values, high steady-state $S_{\mathrm{g}}$ values are established in Experiments 2, 3 and 4 . That difference stems from the lower displacement efficiency (higher mobility) of the weak foam generated in Experiment 1 than in other experiments. Let us then focus on the strong foam displacements. One observes on Figure 13a that an increase of foam quality from 0.4 to $0.7-0.8$ induces an increase of gas saturation of less than $10 \%$ pore volume. Obviously, the relationship between fractional flow and saturation is not straightforward at all in any two-phase displacement. However, a qualitative analysis may be attempted in the present displacement context. It is commonly assumed that foam

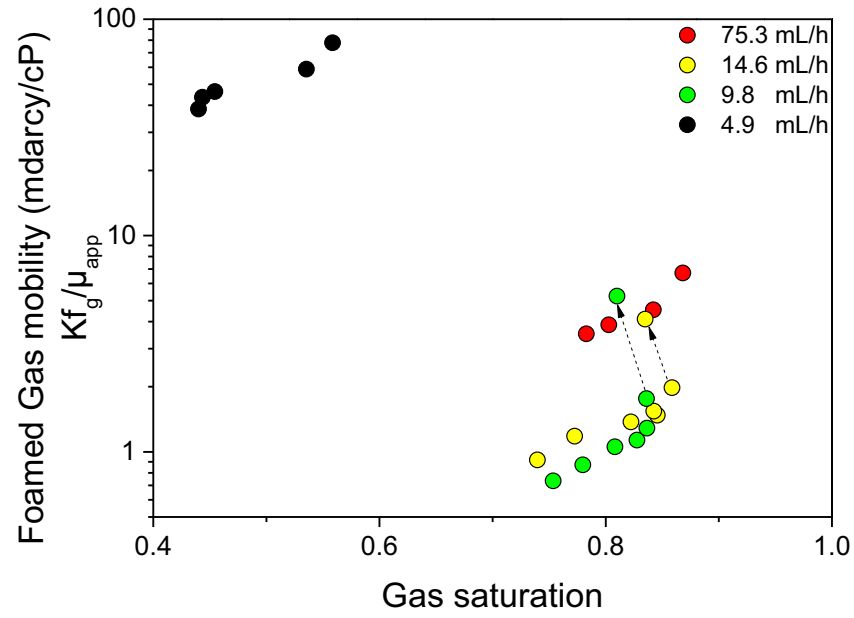

Fig. 12. Gas mobility in foam as a function of gas saturation.

(a)

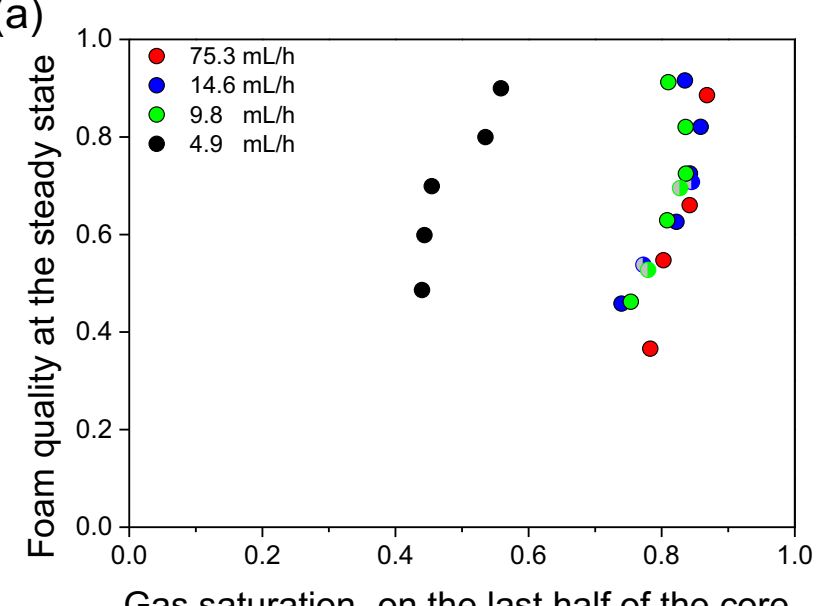

(b)

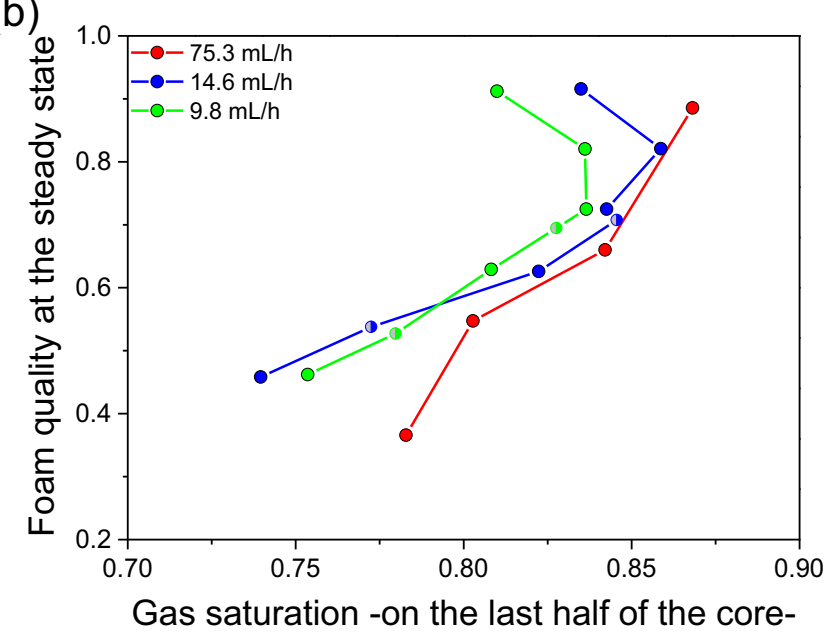

Fig. 13. (a) Foam quality as a function of gas saturation at the steady state (b) Zoom on Experiments 2, 3 and 4. The half colored circles represent the experiments of low-quality foam after reaching the highest foam quality. 


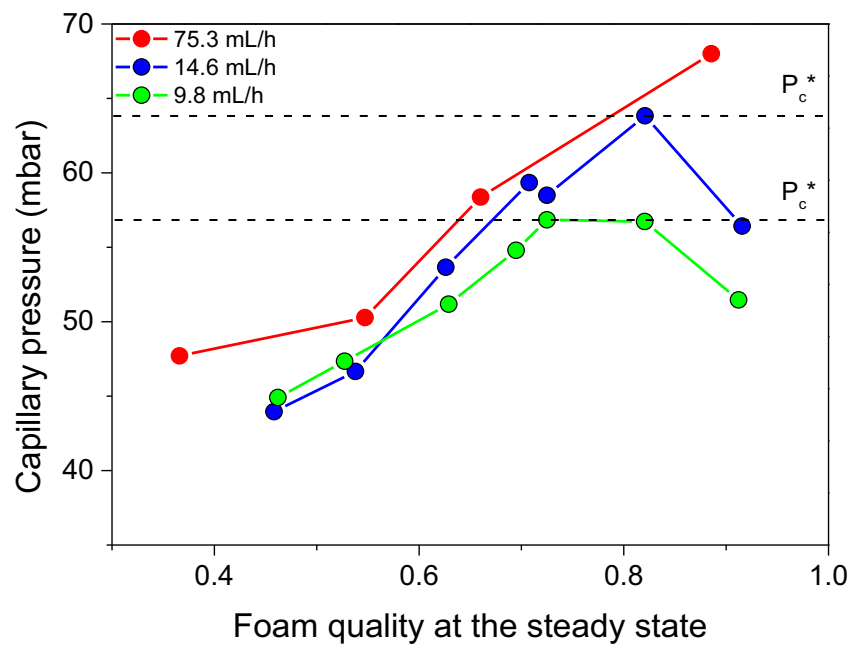

Fig. 14. Capillary pressure as a function of foam quality.

texture, i.e. bubble size, is invariant in the low-quality regime. Therefore, more lamellas are transported by a unit volume of flowing foam when its quality increases. According to Figure 13a, we consider that gas saturation does not change in first approximation. Let us then consider an increase of the foam quality. If the immobile (i.e. trapped) gas saturation does not change, then the mobile gas saturation within the porous medium does not change either according to the constant (total) gas saturation approximation stated at the beginning. Following the invariant bubble size assumption, the number of lamellas per unit volume of flowing gas phase must remain constant, which implies that the apparent foam viscosity should remain constant in the low-quality regime. This inference is consistent with the quasi-constant foam apparent viscosity values determined in the low-quality regime (Fig. 10). Therefore, in first approximation, one may assume that the trapped gas saturation does not change much at steady state when the foam quality increases. In reality, the gas saturation is not strictly invariant and increases slightly as shown in Figure 13b with zoomed saturation scale. That slight gas saturation increase should then be attributed to an increase of the trapped gas saturation.

\subsubsection{Transition to the high-quality regime}

From Figure 13b, we notice an increase of the gas saturation without attaining a maximum at the highest total flow rate $(75.3 \mathrm{~mL} / \mathrm{h})$. A close analysis of results for Experiments 2 and 3 reveals that gas saturation decreases at high-quality values, after reaching a maximum, denoted $S_{\mathrm{g}}^{*}$, for a quality value corresponding to the transition between the low-quality foam regime and the high-quality one. That foam behavior was also found in other experimental studies [53-57]. Kibodeaux and Rossen [55] suggested that different steady-state flows could be established for the same value of $S_{\mathrm{w}}$, thus confirming the non-monotonic behavior of $f_{\mathrm{g}}$ as a function of $S_{\mathrm{g}}$ (or $f_{\mathrm{w}}$ as a function of $S_{\mathrm{w}}$ ). This behavior stems from the collapse of foam that
Table 3. Parameters characterizing the limiting conditions between low and high-quality regimes.

\begin{tabular}{lcc}
\hline Total flow rate $(\mathrm{mL} / \mathrm{h})$ & $S_{\mathrm{g}}^{*}$ & $P_{\mathrm{c}}^{*}(\mathrm{mbar})$ \\
\hline 9.8 & $\sim 0.77$ & $\sim 57$ \\
14.6 & 0.82 & 63.8 \\
75.3 & $\geq 0.88$ & $\geq 68$ \\
\hline
\end{tabular}

causes an increase of foamed-gas mobility, and the latter gives rise to a water re-imbibition of the porous medium.

In order to probe the effect of the porous medium on foam flow, the capillary pressure was read on Pc curve shown in Figure 4 for the gas saturation measured at steady state of each foam injection at given velocity and quality. Results are shown in Figure 14 as a function of the foam quality. For Experiments 2 and 3, the capillary pressure increases as a function of foam quality in the low-quality regime, reaches a maximum at $S_{\mathrm{g}}^{*}$, then decreases in the high-quality regime. That maximum capillary pressure may then be considered as a critical capillary pressure $P_{\mathrm{c}}^{*}=P_{\mathrm{c}}\left(S_{\mathrm{g}}^{*}\right)$. Figure 14 and Table 3 show close values of $P_{\mathrm{c}}^{*}$, however the latter values seem to be reached at different foam qualities depending on the total velocity (Fig. 14). For the highest velocity, $P_{c}^{*}$ would be reached at a higher gas fractional flow than 0.9. At first glance, such a variation of $f_{\mathrm{g}}$ at $P_{\mathrm{c}}^{*}$ seems in contradiction with the unique value of the critical foam quality $f_{\mathrm{g}}^{*}$ (around 0.70) as inferred from steady-state pressure drop values (Fig. 9a). Indeed, the transition from the low-quality to the high-quality regime occurs over a small range of foam qualities rather than for a single value. However, one must be aware of the low sensitivity of gas saturation and of corresponding $P_{\mathrm{c}}$ values to the foam quality. Furthermore, measured pressure drop values refer to the whole core whereas the half downstream part of the core is considered for determining core saturation. Hence, a reliable/sure interpretation of apparent variations of $P_{c}^{*}$ cannot be undertaken.

Beside those experimental limitations, the phenomena underlying $P_{\mathrm{c}}^{*}$ concept are complex. They have been subject of several interpretations $[8,22]$ and some questions remain. For instance, Khatib and co-workers [8] observed a decrease in the limiting capillary pressure when gas flow rate increased. That behavior was interpreted as the result of mechanical disturbances of foam lamellae and was matched by Jimenez and Radke [22] with a model of capillaries having a sinusoidal shape. We did not observe such a decrease of $P_{\mathrm{c}}^{*}$ with gas flow rate increase at a given gas fractional flow from our experimental results. Maybe the minimum velocity for such phenomena to occur has not been reached in our experiments. The porous medium studied herein is also much less permeable than Khatib et al.'s sand or bead packs. Such porous medium differences and the complexity of flow channels in porous media may considerably change foam propagation as suggested by Géraud et al. [32, 33], especially in the coalescence regime. Definitely, observations of foam flow dynamics at the pore scale would enlighten us on that issue. 


\section{Conclusion}

This work aimed at conducting foam flow experiments on a Bentheimer core at different foam qualities and foam velocities coupled with X-ray computed tomography. In-situ saturation profiles gave a valuable reliable insight into the mechanisms of foam generation and propagation taking place within a natural porous medium. The main outcomes are as follows:

(a) Foam flow through the brine-saturated core takes place in two successive phases and reveals a pistonlike displacement pattern of the foam.

(b) Saturation profiles reveal permanent entrance effects and transient downstream end effects:

- Entrance effects are related to the injection procedure, and are attenuated with a high foam quality and/or a low total flow rate.

- Downstream end effects are related to the gradual foam build-up.

- Hence, the use of long cores is recommended as far as possible.

(c) A critical velocity (or pressure gradient) has to be exceeded to generate strong foam, even for a highquality foam.

(d) The evolution of apparent foam viscosity values with foam quality reveals a transition between a lowquality regime and a high-quality regime, respectively characterized by quasi-constant and decreasing apparent viscosity values. Interpreting the quasiinvariance of strong foam apparent viscosity values at fixed total velocity in the low-quality regime requires to assume that increasing foam quality does not change the mobile gas saturation but only increases (slightly) the trapped gas saturation, according to measured (total) gas saturation at steady state.

(e) The foam is a shear-thinning fluid in the low-quality regime. Indeed, foam apparent viscosity values are found to vary as an inverse power function of the total flow velocity.

(f) The critical quality value, above which the pressure drop or the apparent foam viscosity decreases, is related to a given (critical) saturation value of the porous medium (assuming uniform saturation), which can be related to a critical or limiting capillary pressure $P_{\mathrm{c}}^{*}$ marking the onset of the high-quality regime. Analysis of the small differences in the so-determined $P_{c}^{*}$ values cannot be undertaken with regard to the inhomogeneity of flow behavior at core scale and to the complex phenomena involved in the high-quality regime. (g) The main foam flow feature in the high-quality regime is the increase of gas mobility, or the decrease of apparent foam viscosity, with foam quality.

Acknowledgments. The authors thank Marie-Claude Lynch and Fanny Lutz for their help in conducting experiments, and IFPEN for permission to publish this work.

\section{References}

1 Holm L.W. (1970) Foam injection test in the Siggins Field, Illinois, Society of Petroleum Engineers. SPE-2750-PA. doi: 10.2118/2750-PA

2 Jonas T.M., Chou S.I., Vasicek S.L. (eds). (1990) Evaluation of a $\mathrm{CO}_{2}$ foam field trial: Rangely Weber sand unit, Society of Petroleum Engineers.

3 Heller J.P., Boone D.A., Watts R.J. (1985) Field test of $\mathrm{CO}_{2}$ mobility control at Rock Creek, Society of Petroleum Engineers.

4 Hoefner M.L., Evans E.M. (1995) $\mathrm{CO}_{2}$ foam: results from four developmental field trials. SPE-27787-PA. doi: 10.2118/ 27787-PA.

5 Radke C.J., Gillis J.V. (eds) (1990) A dual gas tracer technique for determining trapped gas saturation during steady foam flow in porous media, Society of Petroleum Engineers.

6 Osterloh W.T., Jante M.J. Jr (1992) Effects of gas and liquid velocity on steady-state foam flow at high temperature, Society of Petroleum Engineering.

7 Alvarez J.M., Rivas H.J., Rossen W.R. (eds) (1999) Unified model for steady-state foam behavior at high and low foam qualities, Society of Petroleum Engineers.

8 Khatib Z.I., Hirasaki G.J., Falls A.H. (1988) Effects of capillary pressure on coalescence and phase mobilities in foams flowing through porous media, Society of Petroleum Engineering.

9 Farajzadeh R., Lotfollahi M., Eftekhari A.A., Rossen W.R., Hirasaki G.J.H. (2015) Effect of permeability on implicittexture foam model parameters and the limiting capillary pressure, Energy Fuels 29, 5, 3011-3018. doi: 10.1021/acs. energyfuels.5b00248.

10 Friedmann F., Jensen J.A. (eds) (1986) Some parameters influencing the formation and propagation of foams in porous media, Society of Petroleum Engineers.

11 Kim J., Dong Y., Rossen W.R. (2005) Steady-state flow behavior of $\mathrm{CO}_{2}$ foam. SPE-89351-PA. doi: 10.2118/89351PA.

12 Farajzadeh R., Andrianov A., Bruining H., Zitha P.L.J. (2009) Comparative study of $\mathrm{CO}_{2}$ and $\mathrm{N}_{2}$ foams in porous media at low and high pressure-temperatures, Ind. Eng. Chem. Res. 48, 9, 4542-4552. doi: 10.1021/ie801760u.

13 Farajzadeh R., Muruganathan R.M., Rossen W.R., Krastev R. (2011) Effect of gas type on foam film permeability and its implications for foam flow in porous media, Adv. Colloid Interface Sci. 168, 1-2, 71-78. doi: 10.1016/ j.cis.2011.03.005.

14 Zeng Y., Farajzadeh R., Eftekhari A.A., Vincent-Bonnieu S., Muthuswamy A., Rossen W.R., Hirasaki G.J., Biswal S.L. (2016) Role of gas type on foam transport in porous media, 
Langmuir 32, 25, 6239-6245. doi: 10.1021/acs.langmuir. 6b00949.

15 Vikingstad A.K., Aarra M.G. (2009) Comparing the static and dynamic foam properties of a fluorinated and an alpha olefin sulfonate surfactant, J. Pet. Sci. Eng. 65, 105.

16 Rossen W.R. (1988) Theories of foam mobilization pressure gradient, SPE Enhanced Oil Recovery Symposium, 16-21 April, Tulsa, Oklahoma, Society of Petroleum Engineers.

17 Ashoori E., Marchesin D., Rossen W.R. (2011) Dynamic foam behavior in the entrance region of a porous medium, Colloids Surf. A: Physicochem. Eng. Aspects 377, 1, 217-227. doi: 10.1016/j.colsurfa.2010.12.043.

18 Falls A.H., Musters J.J., Ratulowski J. (1989) The apparent viscosity of foams in homogeneous bead packs, SPE Reserv. Eng. 4, 2, 155-164. doi: 10.2118/16048-PA.

19 Chambers K.T., Radke C.J. (1991) Capillary phenomena in foam flow through porous media, in: Morrow N.R. (ed), Interfacial phenomena in petroleum recovery, Marcel Dekker, New York, NY, pp. 191-256.

20 Lee H.O., Heller J.P., Hoefer A. (1991) Change in apparent viscosity of $\mathrm{CO}_{2}$ foam with rock permeability, SPE Reserv. Eng. 6, 4, 421-428. doi: 10.2118/20194-PA.

21 Zhang Z.F., Zhong L., White M.D., Szecsody J.E. (2012) Experimental investigation of the effective foam viscosity in unsaturated porous media, Vadose Zone J. 11, 4. doi: 10.2136/vzj2011.0190.

22 Jimenez A.I., Radke C.J. (1989) Dynamic stability of foam lamellae flowing through a periodically constricted pore, $A C S$ Symp. Ser. 396, 460-479.

23 Gassara O., Douarche F., Braconnier B., Bourbiaux B. (2017) Calibrating and interpreting implicit-texture models of foam flow through porous media of different permeabilities, J. Pet. Sci. Eng. 159, 588-602. doi: 10.1016/j. petrol.2017.09.069.

24 Hirasaki G.J., Lawson J.B. (1985) Mechanisms of foam flow in porous media: Apparent viscosity in smooth capillaries, Soc. Pet. Eng. J. 25, 2, 176-190. doi: 10.2118/ 12129-PA.

25 Falls A.H., Hirasaki G.J., Patzek T.W., Gauglitz D.A., Miller D.D., Ratulowski T. (1988) Development of a mechanistic foam simulator: the population balance and generation by snap-off, Society of Petroleum Engineers Press. SPE14961-PA. doi: 10.2118/14961-PA.

26 Ma K., Ren G., Mateen K., Morel D., Cordelier P. (2015) Modeling techniques for foam flow in porous media, Society of Petroleum Engineering.

27 Jones S.A., Dollet B., Méheust Y., Cox S.J., Cantat I. (2013) Structure-dependent mobility of a dry aqueous foam flowing along two parallel channels, Phys. Fluids 25, 6, 63101. doi: $10.1063 / 1.4811178$.

28 Kovscek A.R., Radke C.J. (eds) (1994) Fundamentals of foam transport in porous media, ACS Publications, Washington, DC.

29 Rossen W.R., Gauglitz P.A. (1990) Percolation theory of creation and mobilization of foams in porous media, AIChE J. 36, 8, 1176-1188. doi: 10.1002/aic.690360807.

30 Ransohoff T.C., Radke C.J. (1988) Mechanisms of foam generation in glass-bead packs, SPE Reserv. Eng. 3, 2, 573-585. doi: 10.2118/15441-PA.

31 Friedmann F., Chen W.H., Gauglitz P.A. (1991) Experimental and simulation study of high-temperature foam displacement in porous media, Society of Petroleum Engineers. SPE-17357-PA. doi: 10.2118/17357-PA.
32 Géraud B., Méheust Y., Cantat I., Dollet B. (2017) Lamella division in a foam flowing through a two-dimensional porous medium: A model fragmentation process, Phys. Rev. Lett. 118, 9, 98003. doi: 10.1103/PhysRevLett. 118.098003.

33 Géraud B., Jones S.A., Isabelle C., Benjamin D., Yves M. (2016) The flow of a foam in a two-dimensional porous medium, Water Resour. Res. 52, 2, 773-790. doi: 10.1002/2015WR017936.

34 Apaydin O.G., Kovscek A.R. (2001) Surfactant concentration and end effects on foam flow in porous media, Transp. Porous Med. 43, 3, 511-536. doi: 10.1023/A:1010740811277.

35 Nguyen Q.P. (2004) Dynamics of foam in porous media, Doctoral Thesis, TU Delft, The Netherlands.

36 Nguyen Q.P., Currie P.K., Buijse M., Zitha P.L.J. (2007) Mapping of foam mobility in porous media, J. Pet. Sci. Eng. 58, 1-2, 119-132. doi: 10.1016/j.petrol.2006.12.007.

37 Simjoo M., Nguyen Q.P., Zitha P.L.J. (2012) Rheological transition during foam flow in porous media, Ind. Eng. Chem. Res. 51, 30, 10225-10231. doi: 10.1021/ie202218z.

38 Nguyen Q.P., Zitha P.L.J., Currie P.K., Rossen W.R. (eds) (2005) CT study of liquid diversion with foam, Society of Petroleum Engineers.

39 Zitha P.L.J., Nguyen Q.P., Currie P.K. (eds) (2003) Effect of flow velocity and rock layering on foam flow: an X-ray computed tomography study, Society of Petroleum Engineers.

40 Simjoo M., Dong Y., Andrianov A., Talanana M., Zitha P.L. J. (2013) CT scan study of immiscible foam flow in porous media for enhancing oil recovery, Ind. Eng. Chem. Res. 52, 18, 6221-6233. doi: 10.1021/ie300603v.

41 Wellington S.L., Vinegar H.J. (1987) X-ray computerized tomography, J. Pet. Technol., Soc. Pet. Eng. 39, 885-898.

42 Akin S., Kovscek A.R. (2003) Computed tomography in petroleum engineering research, Geol. Soc. Lond. Spec. Publ. 215, 1, 23-38. doi: 10.1144/GSL.SP.2003.215.01.03.

43 Kovscek A.R., Tretheway D.C., Persoff P., Radke C.J. (1995) Foam flow through a transparent rough-walled rock fracture, J. Pet. Sci. Eng. 13, 2, 75-86. doi: 10.1016/09204105(95)00005-3.

44 Gauglitz P.A., Friedmann F., Kam S.I., Rossen W.R. (2002) Foam generation in homogeneous porous media, Chem. Eng. Sci. 57, 19, 4037-4052. doi: 10.1016/S0009-2509(02)00340-8.

45 Tanzil D., Hirasaki G.J., Miller C.A. (eds) (2002) Conditions for foam generation in homogeneous porous media, Society of Petroleum Engineers.

46 Nguyen Q.P., Currie P.K., Zitha P.L.J. (eds) (2003) Determination of foam induced fluid partitioning in porous media using X-ray computed tomography, Society of Petroleum Engineers.

47 Rossen W.R. (1999) Foam generation at layer boundaries in porous media, Society of Petroleum Engineers. SPE-59395PA. doi: 10.2118/59395-PA.

48 Kahrobaei S., Vincent-Bonnieu S., Farajzadeh R. (2017) Experimental study of hysteresis behavior of foam generation in porous media, Sci. Rep. 7, 1, 8986. doi: 10.1038/s41598017-09589-0.

49 Jones S.A., Getrouw N., Vincent-Bonnieu S. (2018) Foam flow in a model porous medium: II. The effect of trapped gas, Soft Matter 14, 18, 3497-3503. doi: 10.1039/c7sm02458d.

50 Rossen W.R., Wang M.W. (1999) Modeling foams for acid diversion, Society of Petroleum Engineers. SPE-56396-PA. doi: 10.2118/56396-PA.

51 Ettinger R.A., Radke C.J. (1992) Influence of texture on steady foam flow in Berea Sandstone, Society of Petroleum Engineering. 
52 Schramm L.L. (1994) Foams: fundamentals and applications in the petroleum industry, American Chemical Society, Washington, DC.

53 Wassmuth F.R., Green K.A., Randall L. (2001) Details of insitu foam propagation exposed with magnetic resonance imaging, Society of Petroleum Engineers. doi: 10.2118/ 71300-PA.

54 Xu Q., Rossen W.R. (2004) Experimental study of gas injection in a surfactant-alternating-gas, Society of Petroleum Engineers. SPE-84183-PA. doi: 10.2118/84183-PA.
55 Kibodeaux K.R., Rossen W.R. (1997) Coreflood study of surfactant-alternating-gas foam processes: implications for field design, Society of Petroleum Engineers.

56 Zhou Z., Rossen W.R. (1995) Applying fractional-flow theory to foam processes at the "limiting capillary pressure", SPE Adv. Technol. Ser. 3, 1, 154-162. doi: 10.2118/24180-PA.

57 Zhou Z.H., Rossen W.R. (1994) Applying fractional-flow theory to foams for diversion in matrix acidization, Society of Petroleum Engineers. SPE-24660-PA. doi: 10.2118/24660PA. 